\title{
DIABETES IS PRIMARY \\ TIMELY NEWS AND NOTES FOR PRIMARY CARE PROVIDERS \\ from the American Diabetes Association
}

\section{IN FOCUS:}

\section{DIGITAL DIABETES LIFESTYLE INTERVENTIONS ..............}

By Max Bingham, PhD

Over the past few months, a series of trials have published results on the effectiveness of diabetes lifestyle interventions that are directed by digitally enabled platforms. Whether they are designed to work through patient portals, electronic health record systems, smartphones, or even text messages, a series of authors suggest that communications with patients on a more intensive basis via technology-driven messaging might result in much better clinical outcomes and-dare we suggest-better attendance at appointments.

And, their results imply that, along with improved health outcomes, the various approaches might be effective across wide geographic areas and many different populations and, indeed, could even overcome language barriers in certain cases.

\section{"Spaced Education" for Diabetes Lifestyle Interventions}

As a case in point, Kerfoot et al. (Diabetes Care, doi. org/cbvc) highlight the use of spaced education and a gaming approach to deliver diabetes self-management education via a mobile app or email.

The approach, they say, is based on two psychological phenomena termed the spacing and testing effects and can, in general terms, improve retention of learning and generate meaningful behavior change. And, applied to diabetes, as these authors did in a clinical trial, they say it can lead to reductions in $\mathrm{A} 1 \mathrm{C}$ over 12 months that are comparable to starting a new diabetes drug.

The approach essentially involves presenting healthrelated scenarios to patients, followed immediately by multiple-choice questions and answers explaining the correct outcomes. The material is then presented again in the following weeks to reinforce learnings and behavior changes.

Max Bingham, PhD, is a science writer and editor in Rotterdam, Netherlands. He can be reached on Twitter at @maxbingham.

https://doi.org/10.2337/cd17-0102

(C)2017 by the American Diabetes Association, Inc.
Reportedly, individuals who played the game for up to 12 months had significant reductions in A1C compared to controls, and further analysis suggested that the biggest improvements occurred in patients with the highest initial levels of A1C. As lead author B. Price Kerfoot told Diabetes Care, "To play, patients only need a desire to improve health, a competitive spirit, and an email account or mobile device."

\section{The Message Is Key in Online Messaging}

The question of whether it is the message, the medium, or the motivation obviously arises with tech-based lifestyle interventions, and according to Chung et al. (Diabetes Care, doi.org/cbvf), it seems that the message is key.

They suggest that secure online messaging, added to routine visits to primary care providers, can improve clinical outcomes and, in particular, improve patients' chances of hitting $\mathrm{A} 1 \mathrm{C}$ targets.

In their analysis of 20,000 patients with type 2 diabetes, they found that, overall, the more patients interacted with their provider via messaging, the better outcomes they had, including hitting A1C targets more frequently.

Additionally, they found that it did not matter whether messaging was initiated by patients or by providers, effectively ruling out motivation as a key factor explaining the effect. As a result, they suggest that such messaging might act as a complementary approach to patient visits to improve outcomes overall.

According to author Sukyung Chung, secure messaging is a convenient route for patients to connect with providers when needed: "We see that messaging did not replace in-person visits, which implies no direct cost saving, but it is reassuring that patients and providers continue to get connected through in-person encounters. Regarding its overall benefit, improved outcomes in all important dimensions of diabetes care quality, with no real cost for patients, speaks for itself." 


\section{Antran Diabetes Association。 \\ Diabetes Is Primary}

IN OTHER NEWS .

\section{Diagnostics: A1C Screening Might Miss Prediabetes and Diabetes}

$\mathrm{A} 1 \mathrm{C}$ is a central measure of diabetes control and one that consistently seems to be in the spotlight regarding its best use. As a case in point, Meijnikman et al. (International Journal of Obesity, http:// doi.org/gbnd46) claims that nearly half of patients who are overweight or obese and who have prediabetes or diabetes would go undiagnosed if screened by $\mathrm{A} 1 \mathrm{C}$ alone as compared to the same testing with an oral glucose tolerance test (OGTT).

The study focused on a Caucasian population of $\sim 1,200$ obese or overweight patients without diagnosed diabetes but considered to be at high risk of developing it. Each participant was tested for glucose tolerance via a series of tests, including fasting plasma glucose (FPG), OGTT, and A1C.

They report that just under $50 \%$ of the population had prediabetes and $\sim 12 \%$ had undiagnosed type 2 diabetes using ADA criteria. However, using just A1C as the only diagnostic test, about half would have been missed for a diagnosis.

The authors concluded, "The diagnostic tests_fasting plasma glucose, [A1C], or OGTT_do not demonstrate complete overlap, and most likely detect different pathophysiological pathways of dysglycemia. Further understanding of which pathophysiological pathway [A1C] reflects in the development of type 2 diabetes is warranted."

continued on p. $197 \rightarrow$ 
continued from p. 195

metabolic health, can be achieved in 12 weeks in patients with advanced type 2 diabetes with something called TeLiPro (Telemedical Lifestyle Intervention Program). Reportedly, the approach combines intensive medical and lifestyle support via telephone, a calorie-restricted diet, self-monitoring of blood glucose, and other measures.

Using a randomized trial, the authors found that the TeLiPro approach led to a decrease in A1C of $1.1 \%$ in 12 weeks, whereas control subjects receiving standard care had an A1C decrease of $0.2 \%$. Significant weight reduction, as well as improvements in many other health measures, reportedly also occurred and were maintained for up to 1 year. They say that the initial calorie restriction likely contributed most to these improvements, although the feedback provided also helped to improve outcomes.

As author Kerstin Kempf told Diabetes Care at the time, "We would see TeLiPro as a quantitatively and economically valuable add-on to the national disease management programs in order to be able to guarantee comprehensive diabetes care in the future."

\section{Do National Programs Need an Electronic Health Approach to Improve Outcomes?}

But what of such national programs? In July, Ely et al. (Diabetes Care http://doi.org/cbvm) reported the outcomes of the first 4 years of implementation of the

ONE TO WATCH: CRISPR-EDITED SKIN GRAFTS TO DELIVER GLUCAGON-LIKE PEPTIDE 1 AND CONTROL BLOOD GLUCOSE IN DIABETES

Skin grafts edited with CRISPR-Cas9 gene technology can reportedly deliver glucagonlike peptide 1 (GLP-1) into circulation and, in the process, control blood glucose and type 2 diabetes-a result likely to be seen as a breakthrough for diabetes.

According to authors Yue et al. (Cell Stem Cell, http://doi.org/cbw4), if the approach can be perfected, it has the potential to deliver many other types of therapeutic proteins, and that means it could have clinical relevance for many diseases in addition to diabetes.

In a series of experiments, the authors initially demonstrate that it is possible to engineer mouse skin cells to produce GLP-1 and, with further editing, to control production via a tetracycline-mediated promotor. The cells could then be cultured in vitro to produce what is essentially graftable skin to then transfer to mice.

After showing that the cells were safe and stable, the authors found that GLP-1 could be stimulated with doxycycline (a tetracycline-based antibiotic). And once they had achieved that, they found that mice with grafts that were fed a high-fat diet developed diabetes and put on weight, but when doxycycline was present in the diet, the mice did not put on weight, had significantly reduced insulin resistance, and their glucose levels normalized.

Although the outcomes are eye-catching, the authors caution that considerable work is needed before the approach could be used in humans with diabetes. Nevertheless, the authors do point out that autologous skin grafts have been used for many years in patients with severe burns, making the approach at least viable in theory.

Commenting in an institutional press release (http://bit.ly/2w1YAxW), author Xiaoyang Wu said, "We didn't cure diabetes, but it does provide a potential long-term and safe approach of using skin epidermal stem cells to help people with diabetes and obesity better maintain their glucose levels." 
Centers for Disease Control and Prevention (CDC)'s National Diabetes Prevention Program (National DPP), and the conclusions were generally positive.

Although the program does not use any particular eHealth (electronic health) or mHealth (mobile health) approach, its lifestyle interventions use a fairly intensive face-to-face weekly approach for the first 6 months, followed by monthly follow-up sessions for an additional 6 months.

Reportedly, the approach can lead to reductions in weight and increases in physical activity, but only if participants maintain adherence to the program. They say that cumulatively, the weight reduction and increased physical activity achieved could have significant effects at the population level.

However, the message is clear: retention and full participation is not actually achieved by the vast majority of participants and that now is the major aim of the program: to get retention rates up.

According to author Elizabeth Ely, "Retention has been a key focus of the National Diabetes Prevention Program and will continue to be. There will be further publications focused on retention strategies and the lessons learned from the large, 4-year evaluation of the National DPP grantees in the coming months."

Could some form of eHealth intervention based on messaging improve outcomes for program participants in the CDC's National DPP, as it has in other programs? We can only assume so. A number of participating centers are already offering online versions of the National DPP curriculum in combination with individual or group access to lifestyle coaches, and it is likely that this will become a norm. Indeed, Fruit Street, a New York-based digital health start-up company, has reportedly received an endorsement from the CDC to combine the National DPP curriculum with their eHealth approach that involves FitBit and other health-related devices (http://bit.ly/2gWkITq).

\section{National Standards for Diabetes Education Support eHealth-Based Interventions}

Individual studies of lifestyle interventions (digital or not) continue to add to the evidence base in support of such interventions to improve outcomes in diabetes. Now, the latest version of the National Standards for Diabetes Self-Management Education and Support (http://doi. org/cbvn) offers a ringing endorsement of lifestyle intervention and particularly digital interventions.

Jointly published at the end of July by the American Diabetes Association and the American Association of Diabetes Educators, the report details a series of standards that providers of diabetes self-management
IN OTHER NEWS

continued from p. 195

\section{And Finally, the Case of the Missing Microbes}

Writing in Nature Reviews Immunology (http:// doi.org/cbvr), Martin Blaser charts out a theory that disappearing microbes and missing microbial taxa in the human microbiome might explain the increased incidence of chronic diseases, including type 1 diabetes and obesity. He explains that early life is a crucial period in which the microbiome becomes established, and cross-talk between the human self and the microbes effectively establishes, through the immune system, what should and should not be present in the body.

Although none of that is particularly new, it is what Blaser presents next that should raise alarm bells clinically. He systematically sets out a series of developments related to modern life that have likely wiped out crucial microbial taxa related to the shaping of human physiology and particularly to the functioning of the immune system.

Central to this theory is the concept of vertical and horizontal transmission of microbes among people and the notion that modern developments may be defeating a process that human health depends on. Caesarean section births, antibiotic use in pregnancy and in early years of life, the use of baby formula, improved sanitation, and even clean water may all be implicated.

Blaser then covers in some detail the likely immunological consequences of the missing microbes. Highlighting studies relating to the role of the microbiome in type 1 diabetes, he suggests that fixing the microbiome might be our only choice to avoid continuing increases in such chronic diseases. He emphasizes that more research will be crucial in developing interventions to repair the microbiome (including better, more specific antibiotics) and that better clinical decision-making is needed to avoid a continued all-out assault on the microbiome. 
continued from p. 197

education and support (DSMES) should follow to ensure that they are offering the highest-quality services for people with diabetes.

Although the DSMES standards focus on the quality of provided care, a patient-centric approach is also highlighted in the latest version. One stand-out point is that the use of technology such as eHealth and mHealth platforms that can improve communications with patients should be widely considered and adopted.

This recommendation is supported by numerous specific recommendations related to tech-enabled support. The key, according to authors of the 2017 DSMES standards report, is that patient data derived from numerous sources should enable a process in which lifestyle intervention programs can be improved over the longer term. Importantly, they say, the feedback these data provide to people with diabetes should enable and encourage them to better manage their diabetes and maintain healthy behavior changes.

\section{Diabetes: There's an App for That}

A gander through the online app stores of Apple and Google will serve up all sorts of solutions that purport to help patients with diabetes. But are any of them any good in the validated, scientific sense? According to Chavez et al. (Diabetes Care, http://doi.org/cbvp), most are a bit thin.

The study they report used a measure of app usability called the Mobile App Rating Scale that is reportedly a validated scoring instrument for rating the quality of mHealth apps. They reportedly identified 120 apps that appear to be related to diabetes and then used an approach akin to the process of developing a systematic review to assess their quality.

The outcome? The authors name just two apps that might be up to scratch in terms of six predefined diabetes management tasks. They were Tactio Health: My Connected Health Logbook and the ACCU-CHEK $360^{\circ}$ Diabetes Management app (since replaced with an updated version called ACCU-CHEK Connect Diabetes Management).

However, the caveats in the study are important to consider. First, the authors only considered free apps and note that some paid-for apps could rate well with the scoring system they used. Second, the study only included apps available up to June 2016. They also point out that the scores the apps received do not necessarily reflect the impact the apps might have in terms of behavior change or health outcomes.

The authors concluded, "This study suggests that additional work is needed to assess the clinical significance of apps for diabetes self-management, and that app developers should work closely with health care providers and patients when building such apps."

\section{Self-Reporting of Patient Data: A Lost Cause?}

And then there is the precautionary tale of Cosson et al. (Diabetes Care, http://doi.org/cbvq). While focused on gestational diabetes, the key message of their study likely applies to diabetes in general: that patients might not report the truth all the time.

Their study focused on 91 women who were given glucose monitoring devices to track their glucose levels during pregnancy and were asked to write down in a notebook what they saw on the screen at specific times. They were told that insulin might be needed if their numbers were outside of a set glucose range, but they were not told that the devices were also recording all their measurements.

In short, they found that what went down in the notebook did not match precisely what was recorded by the devices. The authors rightly sound the alarm that such mismatches could make a huge difference to outcomes.

They say that poor adherence was associated with higher rates of preeclampsia and poor timing of readings was associated with higher $\mathrm{A} 1 \mathrm{C}$ at the time of delivery.

"We could have had even worse results if we had not only included women who had entirely understood how to manage self-monitoring of blood glucose," author Emanuel Cosson told Diabetes Care at the time. "Our results show that ... the memory of blood glucose meters or automatically generated diaries from glucose meters should be used to guide clinical management and also for research purposes." 


\section{POSITIVE CARDIOVASCULAR OUTCOMES REPORTED FOR SEVERAL DIABETES DRUGS}

Data from cardiovascular outcomes trials reported at the $77^{\text {th }}$ American Diabetes Association Scientific Sessions in June showed positive cardiovascular outcomes for several diabetes drugs:

- Results from the DEVOTE trial showed that insulin degludec, an ultra-long-acting, oncedaily basal insulin, does not increase risk for cardiovascular events in subjects with type 2 diabetes at high cardiovascular risk compared to those assigned to insulin glargine treatment. Furthermore, individuals on insulin degludec reported significantly fewer severe and nocturnal hypoglycemia events.

- Results from the CANVAS trial showed that canagliflozin, an SGLT2 inhibitor that works by stimulating the kidneys to expel more glucose in urine, reduces the risk for heart attack, stroke, and cardiovascular event death by $14 \%$ in people with type 2 diabetes. The drug also showed potential kidney benefits. However, canagliflozin was associated with a nearly twofold increased risk for lower limb amputations. This study complements the cardiovascular benefits seen with empagliflozin, another SGLT2 inhibitor drug, supporting the notion that this medication class improves cardiovascular outcomes in people with diabetes who are at high risk of cardiovascular events.

- New analyses from the LEADER trial of the GLP-1 receptor agonist liraglutide affirmed renal benefit and robust cardiovascular benefit for people with diabetes and high cardiovascular risk.

For more highlights from ADA's $77^{\text {th }}$ Scientific Sessions, please access the Summer issue of ADA's DiabetesPro Quarterly at https://professional.diabetes.org/content/diabetes pro-quarterly.

\section{"DIABETES IS PRIMARY" PROGRAM UPDATES PROVIDERS ON THE FRONT LINE OF DIABETES CARE}

For each of the past 6 years, primary care providers from around the world have attended a "Diabetes Is Primary" program offered as a preconference to the American Diabetes Association's annual Scientific Sessions. Developed by the association's Primary Care Advisory Group, the program offers timely updates on diabetes management tailored to primary care providers.

"We know that we can't deliver this practical information to all of primary care in a live session, so we [also] provide webcasts, free of charge," said program vice-chair Eric Johnson, MD, of the Department of Family and Community Medicine at the University of North Dakota School of Medicine \& Health Sciences. "Last year's program distributed more than 10,000 continuing education certificates online, extending the reach of 'Diabetes Is Primary' and sharing this information with other clinicians around the country."

Check out the webcasts and earn up to 5.75 continuing education hours. Visit professional. diabetes.org/ce to learn more. 